\title{
How Should We Study Urban Speciation?
}

\author{
Wouter Halfwerk* \\ Department of Ecological Science, VU University, Amsterdam, Netherlands
}

Keywords: urban speciation, sexual selection, assortative mating, reproductive isolation, mating preferences, signal evolution

\section{INTRODUCTION}

Viewing cities as natural laboratories has great potential to improve our understanding of evolutionary processes. In the past two decades many studies revealed that urban individuals look, sound and behave differently than their non-urban counterparts (reviewed in Sol et al., 2013; Johnson and Munshi-South, 2017; Szulkin et al., 2020). These observations have led to the idea that urbanization can drive speciation and that cities can provide insight into the early stages of this process (Thompson et al., 2018).

Urbanization alters many natural and sexual selection pressures, a theoretical prerequisite for sympatric and parapatric modes of speciation (Kirkpatrick and Ravigné, 2002; van Doorn et al., 2004). Urban phenotypes may therefore represent the start of a new species, an exciting idea that allows us to study the mechanisms involved in the onset of speciation, rather than inferring any early-stage mechanisms from already existing (sub)species pairs. Urban speciation thus has the potential to advance the field of speciation research in general (Butlin et al., 2012). Unfortunately, we lack conclusive evidence of urban speciation at the moment. Many studies have revealed genetic divergence between urban and non-urban populations (reviewed in Johnson and Munshi-South, 2017), however in the face of ongoing geneflow, speciation also requires adaptive divergent selection between these populations (Servedio, 2004; Servedio et al., 2011; Verzijden et al., 2012).

Ecological speciation is the process by which new species form as a consequence of divergent natural or sexual selection pressures between contrasting environments (Nosil et al., 2016). For sexually reproducing organisms this process requires the evolution of assortative mating (= individuals from the same population mating more often than individuals from different populations). Assortative mating can operate via two separate routes: (1) any mechanism that causes individuals from the same population to be in the same location during the same time period; and (2) any mechanism that influences mate choice decisions. The first route is typically caused and influenced by natural selection pressures (e.g., adaptation to the "urban heat island" effect), the second route via sexual selection pressures (e.g., adaptation to increased competition over mates).

Here I would argue that we currently lack conclusive evidence that urban speciation is happening, despite many examples of genetic and phenotypic divergence, as well as some cases of assortative mating. For example, urban and non-urban populations may demonstrate differences in their sexual signals, but whether this divergence influences mating preferences has hardly been tested (Halfwerk et al., 2011). Furthermore, populations may adapt to urban conditions, but whether preferences for locally adapted mates will evolve depends on a genetic linkage between preferences and traits (Kirkpatrick and Ravigné, 2002; Maan and Seehausen, 2011; Butlin et al., 2012). Speciation may even be hampered by natural and sexual selection pressures associated with urbanization (Kirkpatrick and Nuismer, 2004). 
As most of the enthusiasm for urban speciation so far stems from studies that did not explicitly test for any of the crucial assumptions underlying its existence, I therefore call for more direct tests of some of the key components underlying the speciation process that may or may not occur in our cities. Below I discuss what evidence would justify to view cities as natural laboratories to study speciation and provide some examples of most promising study systems to search for it. I advocate that urban speciation most likely occurs and is strongly influenced when the conditions I outline below are met.

\section{URBAN SPECIATION LIKELY FOLLOWS MODELS OF ECOLOGICAL SPECIATION}

Speciation requires populations to become reproductively isolated, which can either be caused by a physical barrier between them (allopatric speciation) or by divergent selection on traits that favor assortative mating. Urban and non-urban populations can experience some level of isolation due to an increase in dispersal barriers, e.g., caused by roads or large areas of impervious surface. Urban populations can therefore evolve from non-urban populations via non-adaptive processes such as drift or founder effects (Perrier et al., 2018; Santangelo et al., 2018; Miles et al., 2019; Rivkin et al., 2019). For example, mosquitos in the London underground tunnels are genetically diverged from their above ground counterparts and this divergence is likely maintained by physical barriers (Byrne and Nichols, 1999). Most urban and non-urban populations would however experience substantial levels of geneflow between them and thus also require a mechanism of assortative mating to set them on the path toward becoming different species.

Ecological speciation models have been widely used to study how reproductive isolation can evolve in the face of ongoing geneflow and how evolutionary processes, such as adaptation, sexual selection, and speciation can influence one and other (Kirkpatrick and Ravigné, 2002; van Doorn et al., 2004; Rundle and Nosil, 2005; Maan and Seehausen, 2011). When trying to understand whether and how urban speciation evolves we can therefore rely to a large extent on theory and predictions derived from these models.

Urban speciation will likely (and probably only) occur under the following three conditions being present (after Kirkpatrick and Ravigné, 2002; Rundle and Nosil, 2005):

(1) ecologically-based divergent selection on traits, (2) reproductive isolation, and (3) a (genetic) mechanism to link trait divergence (1) with isolation (2).

In short, for speciation to occur, urban and non-urban populations should experience divergent selection on specific traits and adaptively respond. The resulting trait divergence than either directly or indirectly initiates and drives assortative mating and thus over time increases reproductive isolation between the two populations. Divergent traits important for mate choice can e.g., directly increase assortative mating (Maan and Seehausen, 2011). Likewise, traits involved in habitat selection, or reproductive timing may directly influence when and where individuals from urban and non-urban populations meet. Adaptive changes to urbanization may also indirectly select for assortative mating. Reproductive isolation may for example evolve as a consequence of selection against nonadapted immigrants, although it is expected that such an indirect process would take more time than direct processes (Plath et al., 2010; Dominoni et al., 2013). Finally, the observed trait divergence and the direct or indirect link to assortative mating requires a mechanism to be maintained, which will most likely be genetic in nature (but see Danchin et al., 2018). As I will briefly review below, in many urban study systems, only one of these conditions has been assessed, or is currently met.

\section{EVIDENCE FOR ECOLOGICALLY-BASED DIVERGENT SELECTION}

An important step in urban speciation events involves divergent selection on traits that are directly or indirectly involved in reproductive isolation between urban and nonurban populations. Biotic and abiotic urban conditions can force populations to adapt specific ecological, phenological, physiological, morphological or behavioral traits. Many urban populations show e.g., higher tolerance to heat stress, suggesting selection has favored specific physiological traits (CampbellStaton et al., 2020). Likewise, changes in predation risk may select for reduced or increased vigilance behavior, whereas changes in food availability may select for traits that alter competitiveness (Valcarcel and Fernández-Juricic, 2009; Halfwerk et al., 2019). Many other examples of adaptive responses to urbanization exist, see e.g., some of the research in this special issue, or some extensive reviews on urban adaptation and evolution (Johnson and Munshi-South, 2017; Szulkin et al., 2020). It is important to keep in mind that many of the observed differences between urban and non-urban individuals may however lack a genetic basis, and may therefore not be the best system (in particular the many vertebrate systems) to look for urban speciation, as not all three conditions might be met (see also below).

\section{DOES ADAPTIVE URBAN EVOLUTION RESULT IN REPRODUCTIVE ISOLATION?}

Adaptive changes in response to urbanization may directly or indirectly select for increased assortative mating between urban and non-urban populations. The direct route either involves divergent sexual selection on traits that function in sexual behavior, such as e.g., signals involved in interand intrasexual communication, or natural selection on traits involved in breeding decisions. Urban individuals could adapt breeding onset to increased temperatures, thereby reducing the temporal overlap with breeding individuals from non-urban populations, as has e.g., been found for blackbirds (Dominoni et al., 2013). Likewise, urban individuals may evolve specific habitat preferences thereby reducing the chance they encounter non-urban individuals. 
Divergent selection on sexual traits mostly stem from studies on birdsong (Slabbekoorn, 2013). Urban acoustic conditions, in particular high levels of traffic sounds favor higher-pitched and louder songs as these suffer from less masking by the noise (Halfwerk and Slabbekoorn, 2009; Kunc and Schmidt, 2019). Many studies have reported urban birds to respond adaptively to noise by sing higher frequency songs, which can in turn directly influence mate choice (Halfwerk et al., 2011; Montague et al., 2013). Most examples involve however immediate signal flexibility, or developmental plasticity, and therefore do not provide the prerequisite of a genetic basis that links trait divergence to mate preferences. Such genetic basis may however evolve through a process of assimilation, assuming populations will maintain their phenotypic differences for long enough periods of time (Servedio, 2004; Danchin et al., 2018). Unfortunately, we lack evidence for assortative mating between urban and non-urban bird populations, as most studies only carried out playback experiments within one population, or used rival responses to song playback as indirect indicator (Ripmeester et al., 2010; Halfwerk et al., 2011). In other words, either the potential for reproductive isolation is unclear, or a genetic basis linking the song variation to this isolation is absent.

Reproductive isolation may also directly evolve via changes in temporal and spatial overlap between two divergent populations. Urban-adapted phenotypes may breed at other times or prefer to breed in specific locations, thereby increasing assortative mating through a simple chance process. Populations can e.g., adapt to the "urban heat island" effect by developing faster or starting to breed earlier (Dominoni et al., 2013). A similar process has already been reported for apple maggot flies which shifted hosts from hawthorn and consequently reduced temporal overlap between populations that inhabit the old host due to higher developmental temperatures in apples (Filchak et al., 2000).

Adaptive changes to urbanization may also indirectly select for assortative mating. Experimental evolution with lab populations suggest that selection for adaptive traits can increase reproductive isolation as a by-product (although tested in the absence of geneflow Rice and Hostert, 1993). Under field conditions, reproductive isolation may also evolve as a consequence of selection against non-adapted immigrants (Nosil et al., 2005). Urban individuals may for example not survive long enough outside the city to reproduce (and non-urban individuals vice versa), although that may seem somewhat extreme given the subtle differences that are often reported in urban studies. Alternatively, species may possess preferences for locally-adapted phenotypes, or evolve these preferences in response to urbanization. Females could for example prefer males that express condition-dependent secondary sexual traits (following a handicap-principle type of sexual selection model; Kirkpatrick and Ryan, 1991). The production of visual pigments involved in signaling is often constrained by a species' diet (e.g., red plumages of many bird species; Svensson and Wong, 2011). Locally-adapted individuals may differ in their feeding performance, influencing their visual displays, which can provide a basis for preference-based assortative mating between urban and non-urban populations. However, most studies focusing on condition-dependent signaling have so far reported that urban phenotypes are duller (Giraudeau et al., 2015).

\section{ADAPTIVE EVOLUTION MAY HAMPER URBAN SPECIATION}

Changes in urban-dependent selection pressures may not always enhance the likelihood of speciation (Candolin, 2009; Halfwerk et al., 2019; Sepp et al., 2020). For example, male tungara frogs (Physaelemus pustulosus) have increased their acoustic signal complexity in urban areas, presumably in response to increased competition over females as well as decreased risk of predation and parasitism (Halfwerk et al., 2019). Such adaptive response to changes in sexual selection can however constrain urban speciation (Kirkpatrick and Nuismer, 2004). In the tungara frog example, urban males have increased signal complexity, but when given a choice, females from both urban and forest populations preferred the urban phenotype. Sexual selection may in this case (and possibly many others) result in asymmetrical instead of decreased geneflow between populations.

\section{DISCUSSION}

In general, (more) studies on divergence in mate preference between urban and non-urban populations are highly needed. Divergent mating preferences may evolve in the absence of variation in the preferred traits, leading to a sensory-exploitation model of sexual selection and speciation (Kirkpatrick and Ryan, 1991). However, mating preferences are most likely to diverge in the presence of sexual trait divergence. In the absence of preference divergence, trait divergence may also lead to assortative mating, but probably only when mate choice is based on phenotypic similarity (e.g., in size, color or time of breeding; Maan and Seehausen, 2011). Assortative mating based on size might be a common mechanism driving speciation in cities. Studies could assess non-random mating in urban vs. non-urban populations based on size (or other traits), followed by mate choice trials in which the similarity trait can be experimentally manipulated (using e.g., video techniques, or robotics). Furthermore, clear cases of adaptive evolution in response to urbanization (e.g., heat tolerance, or adaptations to urban structures) are most prominent to test for the indirect evolution of assortative mating (either through mate choice test under common garden or divergent conditions). Finally, future studies should aim to integrate behavioral and genomic approaches to test for the prerequisite of reproductive isolation and the genetic mechanism(s) linking it to trait divergence, either through common garden breeding experiments to assess heritability, or genome-wide association studies to link genomic regions to divergent traits.

In conclusion, I argue that we currently lack any evidence of urban speciation, despite many examples of sexual behavior being influenced by a life in the city. In part this knowledge gap may be the result of the integrative approach that is required 
to conclusively show that urban and non-urban populations are locally adapted and on the path toward becoming separate species. Furthermore, most of our knowledge from the field comes from long-lived species, such as frogs and birds, that are difficult to track throughout their lives and often show a large extend of behavioral flexibility that can mask any underlying genetic mechanism. Bringing the lab to the field, or the field to the lab may help to unravel the mechanisms involved in urban speciation.

\section{REFERENCES}

Butlin, R., Debelle, A., Kerth, C., Snook, R. R., Beukeboom, L. W., Cajas, R. F. C., et al. (2012). What do we need to know about speciation? Trends Ecol. Evol. 27, 27-39. doi: 10.1016/j.tree.2011.09.002

Byrne, K., and Nichols, R. A. (1999). Culex pipiens in London underground tunnels: differentiation between surface and subterranean populations. Heredity 82, 7-15. doi: 10.1038/sj.hdy.6884120

Campbell-Staton, S. C., Winchell, K. M., Rochette, N. C., Fredette, J., Maayan, I., Schweizer, R. M., et al. (2020). Parallel selection on thermal physiology facilitates repeated adaptation of city lizards to urban heat islands. Nat. Ecol. Evol. 4, 652-658. doi: 10.1038/s41559-020-1131-8

Candolin, U. (2009). Population responses to anthropogenic disturbance: lessons from three-spined sticklebacks Gasterosteus aculeatus in eutrophic habitats. J. Fish Biol. 75, 2108-2121. doi: 10.1111/j.1095-8649.2009. 02405.x

Danchin, E., Nöbel, S., Pocheville, A., Dagaeff, A. C., Demay, L., Alphand, M., et al. (2018). Cultural flies: Conformist social learning in fruitflies predicts long-lasting mate-choice traditions. Science 362, 1025-1030. doi: $10.1126 /$ science.aat1590

Dominoni, D. M., Helm, B., Lehmann, M., Dowse, H. B., and Partecke, J. (2013). Clocks for the city: circadian differences between forest and city songbirds. Proc. R. Soc. B Biol. Sci. 280:20130593. doi: 10.1098/rspb.20 13.0593

Filchak, K. E., Roethele, J. B., and Feder, J. L. (2000). Natural selection and sympatric divergence in the apple maggot Rhagoletis pomonella. Nature 407, 739-742. doi: $10.1038 / 35037578$

Giraudeau, M., Mateos-Gonzalez, F., Cotín, J., Pagani-Nuñez, E., Torné-Noguera, A., and Senar, J. (2015). Metal exposure influences the melanin and carotenoid-based colorations in great tits. Sci. Total Environ. 532, 512-516. doi: 10.1016/j.scitotenv.2015.06.021

Halfwerk, W., Blaas, M., Kramer, L., Hijner, N., Trillo, P. A., Bernal, X. E., et al. (2019). Adaptive changes in sexual signalling in response to urbanization. Nat. Ecol. Evol. 3, 374-380. doi: 10.1038/s41559-018-0751-8

Halfwerk, W., Bot, S., Buikx, J., van der Velde, M., Komdeur, J., ten Cate, C., et al. (2011). Low songs lose potency in urban noise conditions. Proc. Natl. Acad. Sci. U.S.A. 108, 14549-14554. doi: 10.1073/pnas.1109091108

Halfwerk, W., and Slabbekoorn, H. (2009). A behavioural mechanism explaining noise-dependent frequency use in urban birdsong. Anim. Behav. 78, 1301-1307. doi: 10.1016/j.anbehav.2009.09.015

Johnson, M. T. J., and Munshi-South, J. (2017). Evolution of life in urban environments. Science 358:eaam8327. doi: 10.1126/science.aam8327

Kirkpatrick, M., and Nuismer, S. L. (2004). Sexual selection can constrain sympatric speciation. Proc. R. Soc. Lond. Ser. B Biol. Sci. 271, 687-693. doi: $10.1098 /$ rspb.2003.2645

Kirkpatrick, M., and Ravigné, V. (2002). Speciation by natural and sexual selection: models and experiments. Am. Nat. 159, S22-S35. doi: 10.1086/338370

Kirkpatrick, M., and Ryan, M. J. (1991). The evolution of mating preferences and the paradox of the lek. Nature 350, 33-38. doi: 10.1038/350033a0

Kunc, H. P., and Schmidt, R. (2019). The effects of anthropogenic noise on animals: a meta-analysis. Biol. Lett. 15:20190649. doi: 10.1098/rsbl.2019.0649

Maan, M. E., and Seehausen, O. (2011). Ecology, sexual selection, and speciation. Ecol. Lett. 14, 591-602. doi: 10.1111/j.1461-0248.2011.01606.x

\section{AUTHOR CONTRIBUTIONS}

WH conceived and wrote the paper. WH was funded through the ERC-stg project CITISENSE (\#802460).

\section{ACKNOWLEDGMENTS}

I would like to thank Peter Moran for providing valuable comments on an earlier version of this article.

Miles, L. S., Rivkin, L. R., Johnson, M. T. J., Munshi-South, J., and Verrelli, B. C. (2019). Gene flow and genetic drift in urban environments. Mol. Ecol. 28, 4138-4151. doi: $10.1111 / \mathrm{mec} .15221$

Montague, M. J., Danek-Gontard, M., and Kunc, H. P. (2013). Phenotypic plasticity affects the response of a sexually selected trait to anthropogenic noise. Behav. Ecol. 24, 342-348. doi: 10.1093/beheco/ars169

Nosil, P., Comeault, A., and Farkas, T. (2016). "Ecological speciation and its consequences," in Encyclopedia of Evolutionary Biology, ed R. M. Kliman, 487-493. doi: 10.1016/B978-0-12-800049-6.00067-6

Nosil, P., Vines, T. H., and Funk, D. J. (2005). Reproductive isolation caused by natural selection against immigrants from divergent habitats. Evolution 59, 705-719. doi: 10.1111/j.0014-3820.2005.tb01747.x

Perrier, C. A., Lozano del Campo, A., Szulkin, M., Demeyrier, V., Gregoire, A., and Charmantier, A. (2018). Great tits and the city: distribution of genomic diversity and gene-environment associations along an urbanization gradient. Evol. Appl. 11, 593-613. doi: 10.1111/eva.12580

Plath, M., Riesch, R., Oranth, A., Dzienko, J., Karau, N., Schießl, A., et al. (2010). Complementary effect of natural and sexual selection against immigrants maintains differentiation between locally adapted fish. Naturwissenschaften 97, 769-774. doi: 10.1007/s00114-010-0691-x

Rice, W. R., and Hostert, E. E. (1993). Laboratory experiments on speciation: what have we learned in 40 years? Evolution 47, 1637-1653. doi: 10.1111/j.1558-5646.1993.tb01257.x

Ripmeester, E. A. P., Mulder, M., and Slabbekoorn, H. (2010). Habitatdependent acoustic divergence affects playback response in urban and forest populations of the European blackbird. Behav. Ecol. 21, 876-883. doi: 10.1093/beheco/arq075

Rivkin, L. R., Santangelo, J. S., Alberti, M., Aronson, M. F., de Keyzer, C. W., Diamond, S. E., et al. (2019). A roadmap for urban evolutionary ecology. Evol. Appl. 12, 384-398. doi: 10.1111/eva.12734

Rundle, H. D., and Nosil, P. (2005). Ecological speciation. Ecol. Lett. 8, 336-352. doi: 10.1111/j.1461-0248.2004.00715.x

Santangelo, J. S., Rivkin, L. R., and Johnson, M. T. (2018). The evolution of city life. Proc. Biol. Sci. 285:20181529. doi: 10.1098/rspb.2018.1529

Sepp, T., McGraw, K. J., and Giraudeau, M. (2020). "Urban sexual selection," in Urban Evolutionary Biology, eds M. Szulkin, J. MunshiSouth, and A. Charmantier, 234-252. doi: 10.1093/oso/9780198836841.0 03.0014

Servedio, M. R. (2004). The evolution of premating isolation: Local adaptation and natural and sexual selection against hybrids. Evolution 58, 913-924. doi: 10.1111/j.0014-3820.2004.tb00425.x

Servedio, M. R., Van Doorn, G. S., Kopp, M., Frame, A. M., and Nosil, P. (2011). Magic traits in speciation:'magic'but not rare? Trends Ecol. Evol. 26, 389-397. doi: 10.1016/j.tree.2011.04.005

Slabbekoorn, H. (2013). Songs of the city: noise-dependent spectral plasticity in the acoustic phenotype of urban birds. Anim. Behav. 85, 1089-1099. doi: 10.1016/j.anbehav.2013.01.021

Sol, D., Lapiedra, O., and González-Lagos, C. (2013). Behavioural adjustments for a life in the city. Anim. Behav. 85, 1101-1112. doi: 10.1016/j.anbehav.2013.01.023

Svensson, P. A., and Wong, B. (2011). Carotenoid-based signals in behavioural ecology: a review. Behaviour 148, 131-189. doi: 10.1163/000579510X548673

Szulkin, M., Munshi-South, J., and Charmantier, A. (2020). Urban Evolutionary Biology. New York, NY: Oxford University Press. 
Thompson, K. A., Rieseberg, L. H., and Schluter, D. (2018). Speciation and the city. Trends Ecol. Evol. 33.11, 815-826. doi: 10.1016/j.tree.2018.08.007

Valcarcel, A., and Fernández-Juricic, E. (2009). Antipredator strategies of house finches: are urban habitats safe spots from predators even when humans are around? Behav. Ecol. Sociobiol. 63:673. doi: 10.1007/s00265-008-0701-6

van Doorn, G. S., Dieckmann, U., and Weissing, F. J. (2004). Sympatric speciation by sexual selection: a critical reevaluation. Am. Nat. 163, 709-725. doi: $10.1086 / 383619$

Verzijden, M. N., ten Cate, C., Servedio, M. R., Kozak, G. M., Boughman, J. W., and Svensson, E. I. (2012). The impact of learning on sexual selection and speciation. Trends Ecol. Evol. 27, 511-519. doi: 10.1016/j.tree.2012.05.007
Conflict of Interest: The author declares that the research was conducted in the absence of any commercial or financial relationships that could be constructed as a potential conflict of interest.

Copyright (๑) 2021 Halfwerk. This is an open-access article distributed under the terms of the Creative Commons Attribution License (CC BY). The use, distribution or reproduction in other forums is permitted, provided the original author(s) and the copyright owner(s) are credited and that the original publication in this journal is cited, in accordance with accepted academic practice. No use, distribution or reproduction is permitted which does not comply with these terms. 